\title{
Some applications of the Euclidean algorithm
}

\author{
Adhemar Bultheel \\ Marc Van Barel
}

Department of Computer Science,

\author{
K.U.Leuven, \\ Belgium
}




\section{Euclid and Integers}

$$
\begin{aligned}
& r_{-1}=8 r_{0}=6 \\
& 8-6=2 \Longrightarrow \frac{8}{6}=1+\frac{2}{6} \\
& 6-2=4 \\
& 4-2=2 \Longrightarrow \frac{6}{2}=3+\frac{0}{2} \\
& 2-2=0
\end{aligned}
$$

$$
\text { Hence } G C D(8,6)=2
$$$$
\begin{aligned}
& 2=8 \bmod 6 \\
& 0=6 \bmod 2
\end{aligned}
$$

$G C D(a, b)$

$r_{-1}=a, r_{0}=b$

$k=1$

while $r_{k-1} \neq 0$

set $r_{k}=r_{k-2} \bmod r_{k-1}$

$$
k=k+1
$$

endwhile 


\section{Euclid and polynomials}

$$
\begin{gathered}
r_{-1}=x^{4}-x^{3}-x^{2}+3 x+2 \\
r_{0}=x^{3}-3 x^{2}-x-1 \\
G C D\left(r_{-1}, r_{0}\right)=(x-1) \text { because } \\
r_{1}=\left(r_{-1} \bmod r_{0}\right)=2 x^{2}+2 x-4 \\
r_{2}=\left(r_{0} \bmod r_{1}\right)=r_{2}=x-1 \\
r_{3}=\left(r_{1} \bmod r_{2}\right)=0
\end{gathered}
$$




$$
\begin{aligned}
& \text { Continued fractions } \\
& \text { for rational numbers } \\
& \frac{8}{6}=1+\frac{2}{6}=1+\frac{1}{\frac{6}{2}}=1+\frac{1}{3+\frac{0}{2}} \\
& \text { for polynomials } \\
& r_{-1}=x^{4}-x^{3}-x^{2}+3 x+2 \\
& r_{0}=x^{3}-3 x^{2}-x-1 \\
& \frac{r_{-1}}{r_{0}}=(x-2)+\frac{r_{1}}{r_{0}}=(x-2)+\frac{1}{\frac{r_{0}}{r_{1}}} \\
& =(x-2)+\frac{1}{\frac{1}{2} x+\frac{r_{2}}{r_{1}}}=(x-2)+\frac{1}{\frac{1}{2} x+\frac{1}{\frac{r_{1}}{r_{2}}}} \\
& =(x-2)+\frac{1}{\frac{1}{2} x+\frac{1}{(2 x+4)+\frac{0}{x-1}}}
\end{aligned}
$$

Euclid and CF terminates!

- absolute values $\left|r_{k}\right| \searrow$ and $\geq 0$

- degree polynomials $\operatorname{deg} r_{k} \searrow$ and $\geq 0$ 


\section{Euclidean domain}

integral domain with valuation $\partial$

$$
\begin{gathered}
\partial: D \rightarrow \mathbb{N} \\
\partial(a)=0 \text { iff } a=0 \\
\partial(a b) \geq \partial(a), \quad \forall a, b \neq 0 \\
a=b q+r, \quad \partial(r)<\partial(b), \quad b \neq 0
\end{gathered}
$$

In a Euclidean domain GCD defined up to a unit (invertible element)

For $\mathbb{Z}$ : units are $\{1,-1\}$

For $\mathbb{C}[x]$ : units are $\mathbb{C}_{0}$

terminates if $\partial\left(r_{k}\right) \searrow$ and $\geq 0$ 


\section{Never ending story}

$$
\begin{aligned}
& \text { real numbers } \\
& 3.1415926 \ldots=3+\frac{1}{\frac{1}{0.1415926 \ldots}} \\
& =3+\frac{1}{7.0625133 \ldots} \\
& =3+\frac{1}{7+\frac{1}{1}} \\
& 0.0625133 \ldots \\
& =3+\frac{1}{7+\frac{1}{15.9965 \ldots}} \\
& =3+\frac{1}{7+\frac{1}{15+\frac{1}{1+\frac{1}{292.63459 \ldots}}}}
\end{aligned}
$$




\section{Never ending story (part II)}

Laurent series $\mathbb{F}\left\langle z^{-1}\right\rangle$

$$
\pi(z)=3 \cdot z^{0}+1 \cdot z^{-1}+4 \cdot z^{-2}+1 \cdot z^{-3}+\cdots \in \mathbb{F}\left\langle z^{-1}\right\rangle
$$

works with Laurent series as well

$$
\begin{gathered}
\pi(z)=3+\frac{1}{z-4+\frac{1}{\frac{z^{-1}+4 z^{-2}+z^{-3}+5 z^{-4}+\cdots}{15 z^{-2}-z^{-3}+11 z^{-4}+9 z^{-5}+\cdots}}} \\
\text { "quotient" = polynomial part } \\
\text { Laurent series } \mathbb{F}\langle z\rangle
\end{gathered}
$$

"quotient" = principal part + cst term

(Arne Magnus P-fraction) 


\section{Extended Euclid}

Expansion $=$ at each step

$$
\frac{r_{k-2}}{r_{k-1}}=q_{k}+\frac{r_{k}}{r_{k-1}}
$$

$\Downarrow$

$$
\begin{aligned}
& {\left[\begin{array}{ll}
r_{k-1} & r_{k}
\end{array}\right]=\left[\begin{array}{ll}
r_{k-2} & r_{k-1}
\end{array}\right] V_{k} ; \quad V_{k}=\left[\begin{array}{cc}
0 & 1 \\
1 & -q_{k}
\end{array}\right]} \\
& \text { freedom to choose units } \\
& \Downarrow \\
& {\left[\begin{array}{ll}
s_{k} & r_{k}
\end{array}\right]=\left[\begin{array}{ll}
s_{k-1} & r_{k-1}
\end{array}\right] V_{k} ; \quad V_{k}=\left[\begin{array}{cc}
y_{k} & x_{k} \\
c_{k} & a_{k}
\end{array}\right]} \\
& a_{k}=-q_{k} c_{k} ; \quad c_{k}, x_{k} \quad \text { units; } \quad y_{k}=0 \\
& \text { EEA }=\text { keep track of } V_{0} V_{1} \ldots V_{k} \text { as well } \\
& G_{-1}=\left[\begin{array}{cc}
1 & 0 \\
0 & 1 \\
s & r
\end{array}\right] ; \quad G_{k}=G_{-1} V_{1} \ldots V_{k}=\left[\begin{array}{cc}
x_{0 k} & c_{0 k} \\
y_{0 k} & a_{0 k} \\
s_{k} & r_{k}
\end{array}\right]
\end{aligned}
$$




\section{Approximation}

Laurent series $\mathbb{F}\left\langle z^{-1}\right\rangle$

Truncate continued fraction after $k$ steps, gives

$$
-\frac{r_{0}}{s_{0}}=\underbrace{\frac{c_{0 k}}{a_{0 k}}}_{\text {approx }}+\underbrace{\frac{r_{k}}{a_{0 k}}}_{\text {error }}
$$

If $f=-r_{0} / s_{0}$ is strictly proper then

$$
\operatorname{deg}\left(f-\frac{c_{0 k}}{a_{0 k}}\right)=-2 \alpha_{0 k}-\alpha_{k+1}
$$

$\operatorname{deg} a_{k}=\alpha_{k} ; \quad \operatorname{deg} a_{0 k}=\alpha_{0 k}=\alpha_{1}+\cdots+\alpha_{k}$

Minimal partial realization (MPR) problem (Berlekamp-Massey) - (Gragg-Lindquist)

For formal power series $\mathbb{F}[[z]]$

$$
\begin{aligned}
& \text { Padé approximant } \\
& \text { degree } \rightarrow \text { order } \\
& (\mathrm{MPR} \approx \text { Padé at } \infty)
\end{aligned}
$$

Euclid "jumps" over the blocks in the Padé table and progresses along main (sub)diagonal

$\Rightarrow$ notion of minimal Padé approx. (MPA) 


\section{Linear algebra}

$$
\begin{gathered}
f(z)=\sum_{k=1}^{\infty} f_{k} z^{-k} \in \mathbb{F}\left\langle z^{-1}\right\rangle \\
\text { Approximation } \\
f(z) a_{0, k}(z)-c_{0, k}(z)=r_{k}(z)=\mathcal{O}\left(z^{-\alpha_{0, k+1}}\right) \\
\alpha_{0, k+1}=\sum_{i=0}^{k+1} \alpha_{i} ; \quad \alpha_{i}=\operatorname{deg} a_{i} \quad \text { (quotients) }
\end{gathered}
$$

Reformulate with Hankels

$$
\begin{gathered}
{\left[\begin{array}{cccc}
f_{1} & f_{2} & f_{3} & \cdots \\
f_{2} & f_{3} & \cdots & \\
f_{3} & \cdots & & \\
\vdots & & & \\
\vdots & & &
\end{array}\right]\left[\begin{array}{c}
a_{0, k}^{(0)} \\
\vdots \\
a_{0, k}^{\left(\alpha_{0, k}\right)}
\end{array}\right]=\left[\begin{array}{c}
0 \\
\vdots \\
0 \\
\breve{r}_{k} \\
\vdots
\end{array}\right] \leftarrow \alpha_{0, k+1}} \\
H \mathbf{a}_{0, k}=\mathbf{r}_{k}
\end{gathered}
$$

Kernel structure of Hankel matrices 


\title{
Block triangular factorization
}

\author{
$Z=$ downshift operator
}

$$
H\left[Z \mathbf{a}_{0, k}\right]=Z^{T} \mathbf{r}_{k}
$$

Arrange in blocks

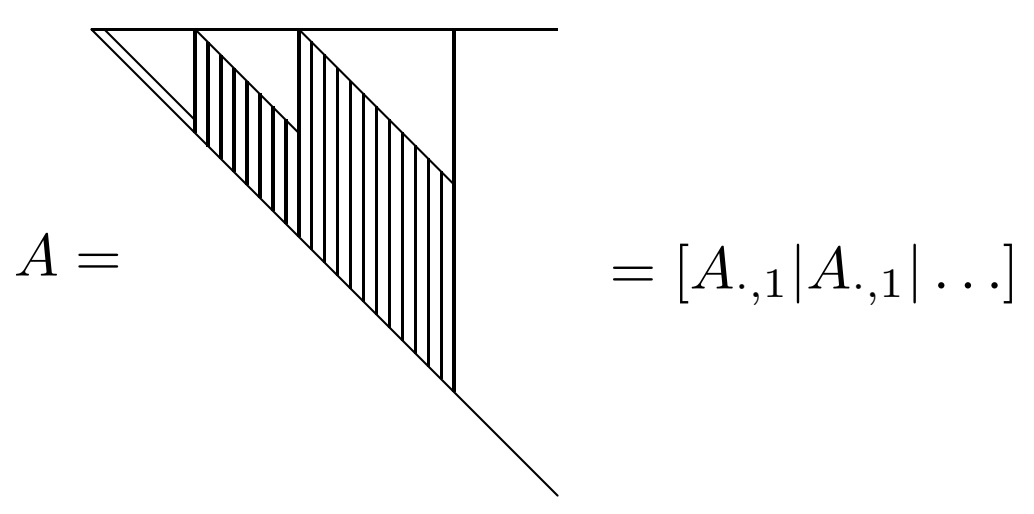

$$
A_{\cdot, k}=\left[\mathbf{a}_{0, k}\left|Z \mathbf{a}_{0, k}\right| Z^{2} \mathbf{a}_{0, k}|\ldots| Z^{\alpha_{k+1}-1} \mathbf{a}_{0, k}\right]
$$

Block triangular factorization

$$
A^{T} H A=\operatorname{diag}\left(D_{0,0}, D_{1,1}, D_{2,2}, \ldots\right)
$$

$$
D_{k, k} \text { AlfACA }^{\alpha_{k+1} \times \alpha_{k+1}}
$$

$$
\begin{gathered}
\alpha_{0, k}=\text { Kronecker indices } \\
\alpha_{k}=\text { Euclidean indices }
\end{gathered}
$$

Leading subHankels $H_{\alpha}$ nonsingular for $\alpha=\alpha_{0, k}$ 's 


\section{Recurrence}

$$
\begin{gathered}
a_{0, k}(z)=a_{0, k-2}(z) \underbrace{x_{k-1} c_{k}}_{\beta_{k}}+a_{0, k-1}(z) a_{k}(z) \\
\left(x_{k-1}, c_{k} \text { units, } a_{k} \text { quotients }\right) \\
\Downarrow
\end{gathered}
$$

Recurrence for blocks

$$
\begin{gathered}
Z A_{, k-1}=A\left[0 \ldots 0\left|T_{k-2, k-1}^{T}\right| T_{k-1, k-1}^{T}\left|T_{k, k-1}^{T}\right| 0 \ldots\right]^{T} \\
T_{k-2, k-1}=\left[\begin{array}{cccc}
0 & \ldots & 0 & -\beta_{k} \\
0 & \ldots & 0 & 0 \\
\vdots & & \vdots & \vdots \\
0 & \ldots & 0 & 0
\end{array}\right] \\
T_{k-1, k-1}=F\left(a_{k}\right)=\left[\begin{array}{cccc}
0 & \ldots & 0 & -a_{k}^{(0)} \\
1 & \ldots & 0 & -a_{k}^{(1)} \\
& \ddots & \vdots & \vdots \\
& & 1 & -a_{k}^{\left(\alpha_{k}-1\right)}
\end{array}\right] \\
T_{k, k-1}=\left[\begin{array}{cccc}
0 & \ldots & 0 & 1 \\
0 & \ldots & 0 & 0 \\
\vdots & & \vdots & \vdots \\
0 & \ldots & 0 & 0
\end{array}\right]
\end{gathered}
$$




\section{Recurrence}

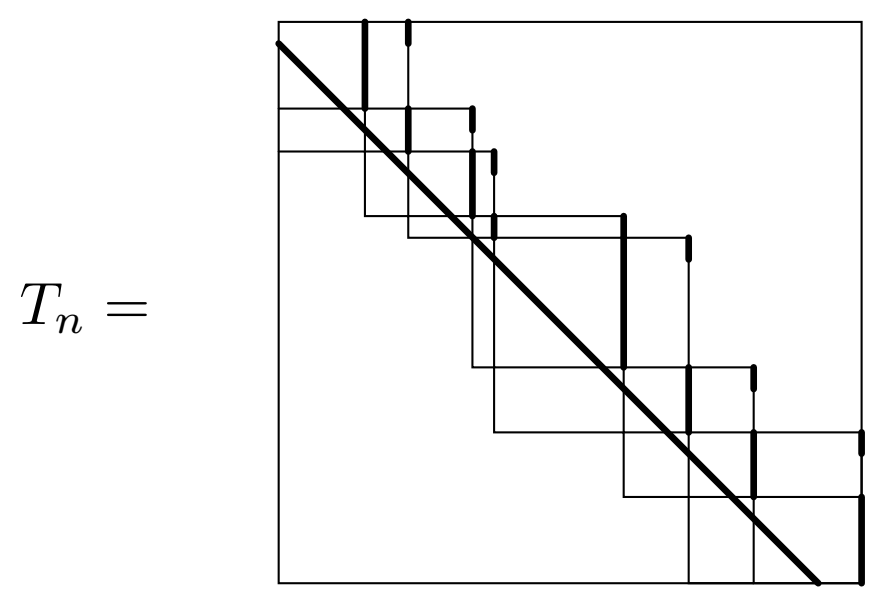

$$
Z A=A T
$$

finite: $F\left(a_{0, n+1}\right) A_{n}=A_{n} T_{n}$

$\operatorname{det} A_{n}=1 \Rightarrow a_{0, n+1}=\operatorname{det}\left(z I-T_{n}\right)$ 


\section{Block orthogonal polynomials}

For finite section of Hankel:

$$
\begin{aligned}
& F\left(a_{0, k+1}\right) A_{k}=A_{k} T_{k} \\
& T_{k}=\left[\begin{array}{cccc}
T_{0,0} & T_{0,1} & & \\
T_{1,0} & T_{1,1} & \ddots & \\
& \ddots & \ddots & \\
& & & T_{k-1, k} \\
& & T_{k, k-1} & T_{k, k}
\end{array}\right]
\end{aligned}
$$

Block 3-term recurrence for formal orthogonal polynomials (Brezinski - Draux)

$A^{T} H A=D$ expresses block orthogonality

$H$ is moment matrix

$Z A=A T$ expresses block 3 -term recurrence

Euclidean algorithm $=$ fast Gram-Schmidt for indefinit metric 


\section{There is more ...}

Adjacent orthogonal polynomials related to shifted moments adjacent diagonal in Padé table

Consider moment matrix $H(z f)$ instead of $H(f)$

$$
A^{T} H(z f) A=D T
$$

( $D$ block diagonal, $T$ block tridiagonal)

$\Downarrow$

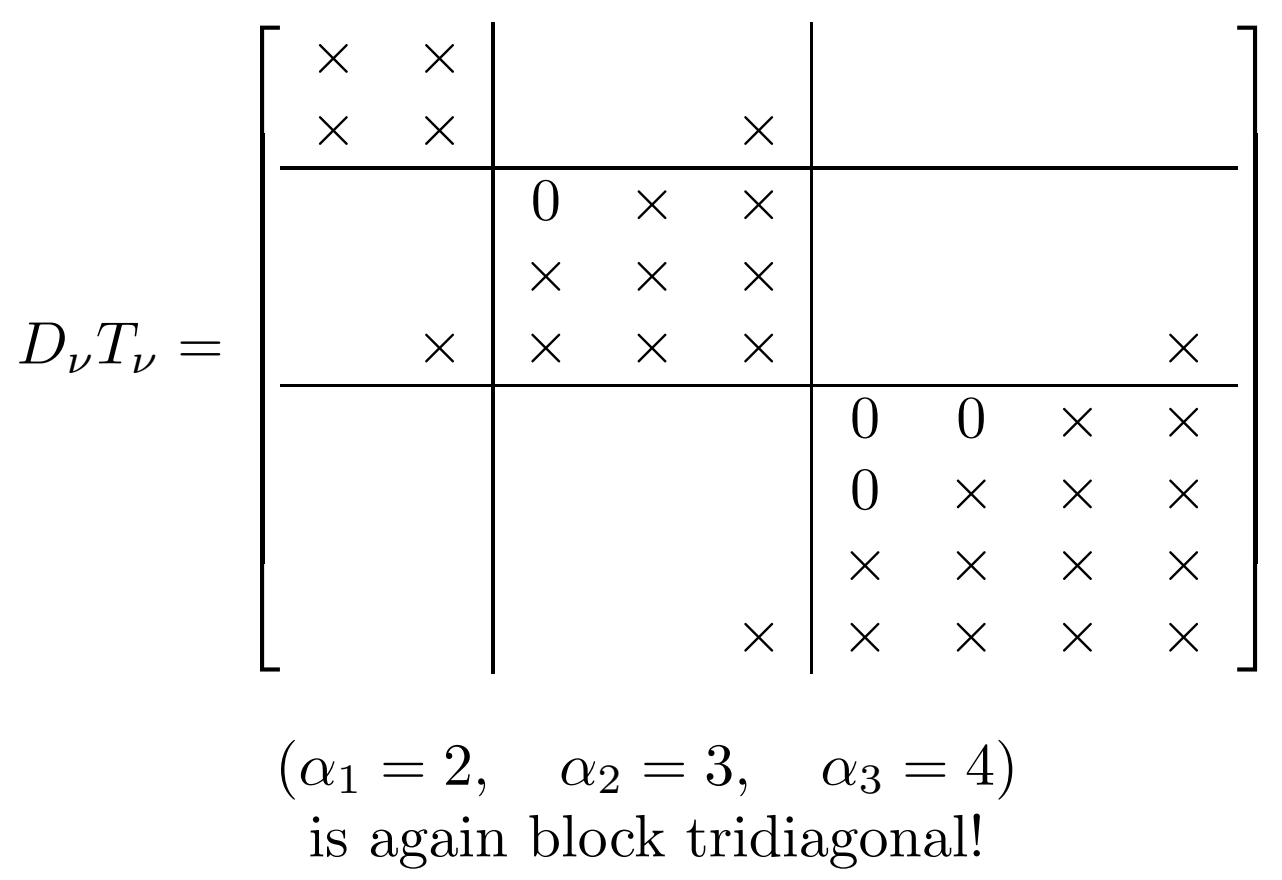




\section{Iterative solution of systems}

$$
\begin{gathered}
\text { system } M x=b \\
\text { residual } r=b-M x \Rightarrow \min \|r\|^{2} \\
\text { conjugate gradient } x_{k}=x_{k-1}+\lambda_{k} s_{k-1} \\
\text { search directions } s_{k}=r_{k}+\mu_{k} s_{k-1} \\
r_{k}=b-M x_{k} \in \mathcal{K}_{n}\left(r_{0}, M\right)=\operatorname{span}\left\{r_{0}, M r_{0}, \ldots, M^{n} r_{0}\right\} \\
\text { Hence } r_{n}=\varphi_{n}(M) r_{0} \\
\text { Suppose } M \text { symmetric } \\
\text { min }\left\|r_{k}\right\|^{2} \Leftrightarrow \min \left\|\varphi_{n}\right\|_{H}^{2} \\
H=\sum(f), \quad f=f_{k} z^{-k}, \quad f_{k}=r_{0}^{H} M^{k+1} r_{0} \\
\text { minimal norm } \Leftrightarrow \varphi_{k} \perp \varphi_{l}, \quad k \neq l \\
\text { Construct OP for Hankel } \equiv \text { supra }
\end{gathered}
$$




\section{Trouble in paradise}

what if $A \neq A^{H}$ and $A \ngtr 0$ ?

$R_{n}^{H} R_{n}$ not Hankel $\Rightarrow$ "long" recurrence

full Gram-Schmidt $\rightarrow$ Arnoldi

GMRES (Saad, 1986)

Why not Hankel?

$$
\begin{array}{r}
r_{i}^{H} r_{j}=r_{0}^{H}\left(A^{H}\right)^{i} A^{j} r_{0} \Rightarrow \text { need } 2 \text { Krylov sequences } \\
\mathcal{K}_{n}\left(x_{0}, A\right) \text { and } \mathcal{K}_{n}\left(y_{0}, A^{H}\right) \\
X_{n}=\left[x_{0}\left|x_{1}\right| \ldots \mid x_{n}\right] \text { and } Y_{n}=\left[y_{0}\left|y_{1}\right| \ldots \mid y_{n}\right]
\end{array}
$$

Hankel again!

$$
Y_{n}^{H} X_{n}=H(f) ; \quad f_{i+j+1}=y_{0}^{H} A^{i} A^{j} x_{0}
$$

Construct

$$
\begin{gathered}
X_{n} \rightarrow \hat{X}_{n}=\left[\hat{x}_{0}|\ldots| \hat{x}_{n}\right] \text { and } Y_{n} \rightarrow \hat{Y}_{n}=\left[\hat{y}_{0}|\ldots| \hat{y}_{n}\right] \\
\hat{Y}_{n}^{H} \hat{X}_{n}=D_{n}(\operatorname{diag}) \\
\hat{Y}_{n}^{H} A \hat{X}_{n}=J_{n} \text { (tridiag) }
\end{gathered}
$$

bi-orthogonalization $\Rightarrow$ BCG (Lanczos, 1950,1952)

two-sided Gram-Schmidt + Krylov = Lanczos

Note $A$ and $J_{n} \approx$ similar $\Rightarrow \lambda(A) \approx \lambda\left(J_{n}\right)$

Can solve eigenvalue problem as well. 


\section{Trouble in paradise (part 2)}

what if $A \neq A^{H}$ and $A \ngtr 0$ ?

Moment matrix $H(f)$ has (nearly) singular submatrices

(near) breakdown (division by zero)

$$
\begin{gathered}
\text { enters Euclid ... (ca 295 BC) } \\
\text { (back to the future) } \\
Y_{n}^{H} X_{n}=H(f) \quad Y_{n}^{H} A X_{n}=H(z f) \\
\hat{X}_{n}=X_{n} A_{n} \quad \hat{Y}_{n}=Y_{n} \bar{A}_{n} \\
\hat{x}_{k}=a_{0, k}(A) x_{0} \quad \hat{y}_{k}=\left[a_{0, k}(A)\right]^{H} y_{0} \\
\hat{Y}_{n}^{H} \hat{X}_{n}=D_{n} \quad \hat{Y}_{n}^{H} A \hat{X}_{n}=D_{n} T_{n} \\
\text { block versions! }
\end{gathered}
$$

Look Ahead Lanczos (LAL) (Parlett-Taylor, 1985)

Quasi Minimal Residuals (QMR) (Freund-Nachtigal, 1991)

Method of Recursive Zoom (MRZ) (Brezinski-M. Redivo Zaglia, 1991) 


\section{Linear systems}

system $\begin{array}{ll}x_{k+1}=A x_{k}+B u_{k}, & A \in \mathbb{F}^{n \times n}, B \in \mathbb{F}^{n \times 1} \\ y_{k+1}=C^{T} x_{k}, & C \in \mathbb{F}^{n \times 1}\end{array}$

state space realization $(A, B, C)$

minimal realization $=n$ is minimal

transfer function $f(z)=C^{T}(z I-A)^{-1} B$

observability matrix $\mathcal{O}=\left[\begin{array}{ll}C & A^{T} C\left(A^{T}\right)^{2} C \ldots\end{array}\right]^{T}$

reachability matrix $\mathcal{R}=\left[\begin{array}{llll}B & A B & A^{2} B & \ldots\end{array}\right]$

observable $n=\operatorname{rank} \mathcal{O}, \quad$ reachable $n=\operatorname{rank} \mathcal{R}$

$$
H=H(f)=\mathcal{O} \mathcal{R}, \quad \operatorname{deg} f(z)=\operatorname{rank} H
$$




\title{
Eigenvalue problems
}

\author{
Here $f(x)=z_{0}^{T}(z I-M) y_{0}$
}

realization $\left(M, y_{0}, z_{0}\right)$

need not be minimal!

size $M=m$

$r_{O}=\operatorname{rank} \mathcal{O}=\operatorname{dim} \mathcal{K}_{\infty}\left(z_{0}, M^{T}\right)$

$r_{R}=\operatorname{rank} \mathcal{R}=\operatorname{dim} \mathcal{K}_{\infty}\left(y_{0}, M\right)$

$r=\operatorname{deg} f=\operatorname{rank} H=\operatorname{rank} \mathcal{O R}$

Spectral info only from $f$

When exact fit of $f: \alpha_{0, n+1}=\operatorname{deg} f<m$

zeros of $a_{0, n+1}(z)$ in spectrum of $M$

$m>r=\min \left\{r_{O}, r_{R}\right\} \quad$ reachable or observable recover spectrum of $M$ restricted to $\mathcal{K}_{\infty}\left(z_{0}, M^{T}\right)$ or $\mathcal{K}_{\infty}\left(y_{0}, M\right)$

$r<\min \left\{r_{O}, r_{R}\right\} \quad y_{0}$ and/or $z_{0}$ are bad no components along max Jordan blocks recover spectrum of $M$ restricted to subspace. 


\section{Error Correcting Codes}

Symbols

$G F(q)=\{0,1, \ldots, q-1\} \quad(\bmod q$ arithmetic $)$

Words

$V_{n}(G F(q))=\left\{\left(\alpha_{0}, \ldots, \alpha_{n-1}\right) ; \alpha_{i} \in F_{q}\right\}$

$(n, k)$-Code defined by set of code words $C$

$$
C \subset V_{n}(G F(q)) ; \quad \operatorname{dim} C=k
$$

Goppa Code defines $C$ as

$G$ generator polyn. over $G F\left(q^{m}\right) ; \quad \operatorname{deg} G=s$

$$
\begin{gathered}
\left\{a_{0}, \ldots, a_{n-1}\right\} \subset G F\left(q^{m}\right) \text { and } G\left(a_{i}\right) \neq 0 \\
\left(\alpha_{0}, \ldots, \alpha_{n-1}\right) \in C \Leftrightarrow S(x)=\sum_{i=0}^{n-1} \frac{\alpha_{i}}{x-a_{i}} \equiv 0(\bmod G(x)) \\
S(x) \text { is syndrome }
\end{gathered}
$$

$$
\mathrm{BCH}: q=2 ; n=2^{m}-1 ; G(x)=x^{2 t}
$$

Reed-Solomon : $n=q^{m}-1 ; G(x)=\prod_{k=1}^{2 t}\left(x-\alpha^{k}\right)$ 


\section{Coding and Euclid}

$$
\begin{aligned}
& \text { transmit } c=\left(c_{0}, \ldots, c_{n-1}\right) \\
& \text { receive } r=\left(r_{0}, \ldots, r_{n-1}\right) \\
& \text { error } e=r-c=\left(e_{0}, \ldots, e_{n-1}\right) \\
& \text { syndrome } \\
& S(x)=\sum_{i=0}^{n-1} \frac{r_{i}}{x-a_{i}}(\bmod G)=\sum_{i=0}^{n-1} \frac{e_{i}}{x-a_{i}}(\bmod G) \\
& E=\left\{a_{i}: e_{i} \neq 0\right\} \\
& \text { location: } \pi(x)=\prod_{\beta \in E}(x-\beta) \\
& \text { amount: } \omega(x) \text { interpolates } \omega\left(\alpha_{i}\right)=e_{i} \\
& \pi(x) S(x)=\omega(x)(\bmod G(x)) \\
& (G, S) \rightarrow(\pi, \omega) \\
& G_{-1}=\left[\begin{array}{cc}
1 & 0 \\
0 & 1 \\
G & S
\end{array}\right] ; \quad G_{k}=G_{-1} V_{1} \ldots V_{k}=\left[\begin{array}{cc}
x_{0 k} & c_{0 k} \\
\pi(x) & a_{0 k} \\
\omega(x) & r_{k}
\end{array}\right]
\end{aligned}
$$




\section{Layer peeling/adjoining}

$$
\begin{gathered}
\text { Berlekamp-Massey = layer adjoining } \\
\text { Euclid = layer peeling }
\end{gathered}
$$

EA : $\quad$ recurrence on $r_{k}($ tails $\rightarrow R$ )

peels off one term in $\mathrm{CF}$

continues with tail

BMA : recurrence on $a_{0 k}$ (denominators $\rightarrow A$ )

adds one term in $\mathrm{CF}$ to $a_{0 k}$

necessary elements of $r_{k}$ for next step

$$
H A=R
$$

BMA : necessary elements from $R$ as inner products.

bottleneck for parallel implementation

$$
\text { EEA }=\text { natural compromise }
$$




\section{Generalizations}

$$
\begin{aligned}
& \text { Matrix versions } \\
& \text { Replace } \mathbb{F} \text { by } \mathbb{F}^{q \times p} \\
& \underline{\text { linear systems }} \\
& (A, B, C): \quad A \in \mathbb{F}^{n \times n}, \quad B \in \mathbb{F}^{n \times p}, \quad C \in \mathbb{F}^{n \times q} \\
& f(z)=s(z)^{-1} r(z), s(z) \in \mathbb{F}^{q \times q}[z], r(z) \in \mathbb{F}^{q \times r}[z] \\
& \underline{g=G C L D(s, r)} \begin{cases}\text { CLD: } & \exists \tilde{s}, \tilde{r}: s=g \tilde{s}, \quad r=g \tilde{r} \\
\text { Gcld: } \quad \forall \tilde{g}=C L D(s, r): \exists h: g=\tilde{g} h\end{cases} \\
& \text { property: } g=G C L D(s, r) \text { if }[s r] V=\left[\begin{array}{ll}
g & 0
\end{array}\right] \\
& V=\text { product of unimod Gaussian elimination matrices } \\
& -s_{0}^{-1} r_{0}=\frac{y_{0}\left(c_{1}+y_{1} \frac{c_{2}+y_{2} \frac{\cdots}{\cdots}}{a_{2}+x_{2} \frac{\cdots}{\cdots}}\right)}{a_{0}\left(a_{1}+x_{1} \frac{c_{2}+y_{2} \frac{\cdots}{\cdots}}{a_{2}+x_{2} \frac{\cdots}{\cdots}}\right)} ; \text { where } \frac{a}{b}=a b^{-1}
\end{aligned}
$$

\section{$\underline{\text { continued fractions }}$}

convergents $=$ successive approximants 


\title{
Generalizations (cntd)
}

\author{
approximation of series
}

left-right duality, matrix Padé approx,...

general rational interpolation, ...

vector Padé : type I - type II duality

$$
\begin{gathered}
\sum_{k} p_{k}(z) f_{k}(z)=O\left(z^{\omega}\right) \\
f_{k}(z) p_{0}(z)-p_{k}(z)=O\left(z^{\omega}\right)
\end{gathered}
$$

$\approx$

rational approx - lin. Diophantine eqs downward sloping - upward sloping diag.

rational approx - GCD 


\section{Look-ahead}

\section{$\mathrm{EEA}=$ avoid breakdown \\ SEEA $=$ avoid near-breakdown}

$\mathrm{EEA}=$ jump from regular point to next one

$\mathrm{SEEA}=$ jump from well conditioned pt to next one

same idea of superfast alg. by divide+conquer

$$
V_{1 n}=\underbrace{V_{1} \ldots V_{k}}_{V_{1}^{s}} \underbrace{V_{k+1} \ldots V_{k+l}}_{V_{2}^{s}} \cdots \underbrace{V_{n-j} \ldots V_{n}}_{V_{t}^{s}}
$$

applied in Lanczos, Padé approx., rational interpol., solution of Hankel and Toeplitz systems, ...

can be applied to follow arbitrary path

$$
V_{k}^{s}=\left[\begin{array}{ll}
y_{k} & c_{k} \\
x_{k} & a_{k}
\end{array}\right]
$$

but $y_{k}, x_{k}, c_{k}, a_{k}$ all polynomials, $V_{k}^{s}$ unimodular

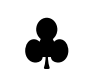

latest challenge : time variant systems : $\left(A_{k}, B_{k}, C_{k}\right)$ 